\title{
ER Bodies Are Induced by Pseudomonas syringae and Negatively Regulate Immunity
}

\author{
José S. Rufián ${ }^{1,2,3}$, James M. Elmore ${ }^{2}$, Eduardo R. Bejarano ${ }^{1}$, Carmen R. Beuzon ${ }^{1}$, and Gitta L. Coaker ${ }^{2, \dagger}$ \\ ${ }^{1}$ Instituto de Hortofruticultura Subtropical y Mediterránea, Universidad de Málaga-Consejo Superior de Investigaciones \\ Científicas (IHSM-UMA-CSIC), Dept. Biología Celular, Genética y Fisiología, Campus de Teatinos, Málaga E-29071, Spain \\ ${ }^{2}$ Department of Plant Pathology, University of California Davis, Davis, CA 95616, U.S.A. \\ ${ }^{3}$ Shanghai Center for Plant Stress Biology, CAS Center for Excellence in Molecular Plant Sciences; Chinese Academy of \\ Sciences, Shanghai 201602, China
}

Accepted 7 May 2021.

\begin{abstract}
ER bodies are endoplasmic reticulum-derived organelles present in plants belonging to the Brassicales order. In Arabidopsis thaliana, ER bodies are ubiquitous in cotyledons and roots and are present only in certain cell types in rosette leaves. However, both wounding and jasmonic acid treatment induce the formation of ER bodies in leaves. Formation of this structure is dependent on the transcription factor NAII. The main components of the ER bodies are $\beta$-glucosidases (BGLUs), enzymes that hydrolyze specialized compounds. In Arabidopsis, PYK10 (BGLU23) and BGLU18 are the most abundant ER body proteins. In this work, we found that ER bodies are downregulated as a consequence of the immune responses induced by bacterial flagellin perception. Arabidopsis mutants defective in ER body formation show enhanced responses upon flagellin perception and enhanced resistance to bacterial infections. Furthermore, the bacterial toxin coronatine induces the formation of de novo ER bodies in leaves and its virulence function is partially dependent on this structure. Finally, we show that performance of the polyphagous beet armyworm herbivore Spodoptera exigua increases in plants lacking ER bodies. Altogether, we provide new evidence for the role of the ER bodies in plant immune responses.
\end{abstract}

Keywords: ER bodies, plant immunity, Pseudomonas syringae, Spodoptera exigua

Current address for James M. Elmore: Department of Plant Pathology and Microbiology, Iowa State University, Ames, IA 5001, U.S.A.

${ }^{\dagger}$ Corresponding author: G. Coaker: glcoaker@ucdavis.edu

Funding: J. S. Rufián has been supported by a FPI fellowship associated with a grant to E. R. Bejarano (Ministerio de Ciencia e Innovación [MICINN], Spain: AGL2010-22287-C02-2), funds from BIO2012-35641 (MICINN, Spain) granted to C. R. Beuzón, and Plan Propio de la Universidad de Málaga-Andalucía Tech. The work was co-funded by European Regional Development Funds (FEDER). G. Coaker is supported by grants from the National Institute of General Medical Sciences (RO1GM092772, R35GM13640).

*The $e$-Xtra logo stands for "electronic extra" and indicates there are supplementary materials published online.

The author(s) declare no conflict of interest.

(c) (1) () $\odot$ Copyright $(92021$ The Author(s). This is an open access article distributed under the CC BY-NC-ND 4.0 International license.
ER bodies are rod-shaped endoplasmic reticulum (ER)-associated structures that are present in three families within the Brassicales order (Nakano et al. 2014). ER bodies are constitutively present in roots and cotyledons of young plants (constitutive ER bodies), but their abundance decreases with senescence (Matsushima et al. 2002). In Arabidopsis rosette leaves, ER bodies are constitutively present in marginal cells, epidermal cells covering the midrib, and extremely large pavement cells (leaf ER bodies) (Nakazaki et al. 2019b). Furthermore, the formation of ER bodies in rosette leaves is induced by methyl jasmonate (MeJA) treatment or wounding (inducible ER bodies) (Matsushima et al. 2002).

Formation of both constitutive and leaf ER bodies is dependent on the basic helix-loop-helix transcription factor NAII (Matsushima et al. 2004) and the essential protein NAI2 (Yamada et al. 2008). Inducible ER bodies are formed in a nail mutant but differ in shape and composition compared with the ER bodies induced in wildtype Col-0 (Ogasawara et al. 2009). The main components of the constitutive ER bodies are the $\beta$-glucosidases (BGLU) PYK10 (BGLU23), BGLU21, and BGLU22 (Matsushima et al. 2003), while inducible-ER bodies also contain BGLU18 (Ogasawara et al. 2009). These myrosinases belong to a subfamily of eight $\beta$-glucosidases (i.e., BGLU18 to BGLU25) (Xu et al. 2004) with ER-retention signals (Yamada et al. 2011). Leaf ER bodies mainly contain PYK10 and BGLU18 (Nakazaki et al. 2019b). PYK10 has been described as an atypical myrosinase that hydrolyzes indole glucosinolates (IG) (Nakano et al. 2017). Upon tissue damage, PYK10 associates in the cytosol with PYK10-binding protein 1 (PBP1), activating PYK10 function (Nagano et al. 2005). An Arabidopsis pyk10/blgul8 double mutant accumulates high amounts of the IG 4-methoxyindol-3-ylmethylglucosinolate (4MI3G) after tissue damage (Nakazaki et al. 2019b). IGs and the products resulting from their breakdown have a role in defense responses against microbial pathogens and herbivory (Bednarek et al. 2009; Clay et al. 2009; Johansson et al. 2014; Millet et al. 2010; Müller et al. 2010; Nakazaki et al. 2019b). IGs are also required for the establishment of beneficial interactions with the endophytic fungus Piriformospora indica by appropriately regulating the level of root colonization through PYK10 (Sherameti et al. 2008; Lahrmann et al. 2015). Collectively, multiple independent studies have supported a role in defense responses for ER bodies (Yamada et al. 2011; Nakano et al. 2014).

Plants are constantly exposed to a variety of biotic and abiotic stresses. Surface-localized plant-pattern recognition receptors (PRRs) act as one of the first lines of defense against pathogens and are able to recognize conserved pathogen features, such as 
flagellin, as non-self (Couto and Zipfel 2016). One of the bestcharacterized PRRs is the flagellin receptor FLS2 (flagellin sensing 2), which recognizes flg22, a 22-amino acid epitope of bacterial flagellin (Gómez-Gómez and Boller 2000). Perception of flg22 by FLS2 induces a number of cellular events resulting in pattern-triggered immunity (PTI), which restricts pathogen proliferation. Some of the cellular events that take place during PTI are the activation of mitogen-activated protein kinases (MAPKs) and calcium-dependent protein kinases, the production of reactive oxygen species (ROS), stomatal closure, and the formation of callose ( $\beta$-1,3-glucan) deposits in the cell wall (Henry et al. 2013). However, successful pathogens have evolved different mechanisms to suppress defense responses and successfully colonize their host.

Pseudomonas syringae is a gram-negative bacterium that has been extensively used to study plant immune signaling. $P$. syringae virulence depends on the type III secretion system (T3SS), a complex nanomachine that translocates proteins, called effectors, into the cytosol of the plant cell. Most of the effectors suppress PTI acting on different cellular pathways (Macho 2016). Another virulence mechanism used by bacterial pathogens to subvert plant immunity is the production of phytotoxins. P. syringae pv. tomato DC3000 produces coronatine, a polyketide toxin that acts as a molecular mimic of the plant hormone jasmonic acid (JA) (Weiler et al. 1994). Coronatine induces stomata reopening upon PRR perception to allow bacterial colonization (Melotto et al. 2006, 2008; Gimenez-Ibanez et al. 2017) and also suppresses callose deposition and induces bacterial growth within the apoplast (Geng et al. 2012).

In this work, we investigated the biological function of ER bodies during plant immunity. We found that ER bodies are downregulated after flg22-triggered immune activation but are induced after $P$. syringae infection in a coronatine-dependent manner. Furthermore, loci underlying ER body formation act as negative regulators of immunity against bacterial pathogens but are required for a complete response against the chewing insect Spodoptera exigua. With these results, we report a new role of ER bodies in virulence of phytopathogenic bacteria and their suppression by plant immune perception.

\section{RESULTS}

\section{ER bodies are rapidly inhibited upon flagellin perception.}

To investigate the role of ER body formation after plant pathogen perception, we used an Arabidopsis transgenic line expressing green fluorescent protein with the ER-retention signal HDEL (GFP-HDEL), which allows the visualization of ER bodies (Mitsuhashi et al. 2000). We vacuum-infiltrated 2-week-old Arabidopsis plants with either $100 \mathrm{nM}$ flg22 or water and visualized cotyledons $3 \mathrm{~h}$ later by confocal microscopy. We were able to detect and quantify the ER bodies in cotyledons, in which this structure is constitutively present, in mock-treated plants. However, after flg22 treatment, only a small number of ER bodies were detected (Fig. 1A). This decrease in ER body detection was statistically different compared with water-treated controls (Fig. 1A). To further investigate the disappearance of ER bodies within the same tissue, we monitored cotyledons for $120 \mathrm{~min}$ after either water (mock) or flg22 treatment. ER bodies lose their characteristic rod shape within 60 min post flg22 treatment, and the GFP signal is diffused throughout the cell at later timepoints (Fig. 1B).

To analyze the impact of the flg22 treatment in ER body formation at the transcriptional level, we analyzed the transcriptional expression of NAII and PYKIO under different biotic treatments, using an available dataset (NASCArrays-120). We found that flg22 treatment reduces the expression level of NAII and PYK10 in an FLS2-dependent manner (Fig. 1C; Supplementary Fig. S1) (Bjornson et al. 2021), pointing to regulation of ER bodies at the transcriptional level as a consequence of activation of defense responses. However, inoculation with the virulent bacterial strain $P$. syringae pv. tomato DC3000 induces the expression of both NAII and PYK1O at $24 \mathrm{~h}$ and the same occurs with a strain expressing the avirulence effector AvrRpm1 (Fig. 1C). Interestingly, both the T3SS DC3000 mutant derivative $\Delta h r c C$ and the nonhost strain 1448A reduced the accumulation of NAII transcripts (Fig. 1C). Altogether, these results show a downregulation of ER bodies as a consequence of the activation of flg22-triggered immunity and suggest a role of this subcellular structure during the defense response against $P$. syringae.

\section{Arabidopsis mutants impacting ER body formation and contents exhibit enhanced immune responses against bacterial pathogens.}

After flg22 perception, many cellular events take place to restrict pathogen invasion, including activation of MAPKs and calcium dependent protein kinases, minutes after perception, followed by ROS production, activation of defense gene expression, and, several hours later, callose deposition (Henry et al. 2013). To further characterize the role of the ER bodies in flg22-induced responses, we measured ROS production as an early event of PTI in plants affected in the formation of ER bodies. We treated 3-week-old Col-0, nail-1, pyk10-1, and pyk10-1/blgu21 plants with $100 \mathrm{nM}$ flg22 and measured the ROS burst. The total ROS produced in all the mutant backgrounds tested was higher than the ROS produced in Col-0 (Fig. 2A). This increase in ROS production in response to flg22 also occurs in both bglu18-1 and pbp1-1 mutants (Supplementary Fig. S2A). To analyze a late event of PTI, we treated 4-week-old plants with flg22 and quantified callose deposition. Consistent with the ROS burst, the number of callose deposits was two times higher in ER body mutants compared with Col-0 (Fig. 2B). These data indicate that a loss of ER bodies or their main components induce an enhanced response to bacterial flg22, pointing to a negative role of ER bodies on PTI.

To determine the role of the ER bodies in bacterial growth, we quantified $P$. syringae pv. tomato DC3000 titers in Col-0 and in the different mutant backgrounds (Fig. 2C). P. syringae pv. tomato DC3000 exhibited decreased growth $(>1 \log )$ in the nail-1 mutant compared with Col-0. Interestingly, both the pyk10-1 and the pyk10-1/bglu21 mutants exhibited a stronger decrease in bacterial growth, with $1.5 \mathrm{log}$ reduction compared with wild-type Col-0 (Fig. 2C). No differences were detected between pyk10 and pyk10-1/bglu21, indicating that $P Y K 10$ is a critical gene involved in negative regulation of bacterial growth. Plants lacking $P B P 1$, necessary for PYK10 function, or BGLU18, the main component of inducible ER bodies, also showed enhanced resistance to bacterial infection (Supplementary Fig. S2B).

A critical component controlling bacterial virulence is the ability to deliver effectors through the T3SS (Macho 2016). In order to investigate the relationship between immune suppression and ER body formation, we used DC3000 $\Delta h r c C$, which is unable to translocate effectors to the plant cell and, thus, does not suppress PTI. We measured the growth of $\Delta h r c C$ in ER body mutants. In the nail-1 background, the growth of $\Delta h r c C$ was not significantly different from that in Col-0. However, we observed a slight but significant decrease in growth in the pyk10-1/bglu21 background (Fig. 2D). The increased $P$. syringae growth restriction in pyk10-1, and pyk10-1/bglu21 backgrounds compared with nail (Fig. 2C and D) could indicate a NAI1-independent regulation for one or both PYK10 and BGLU21. Altogether, these results show that the absence of ER bodies increases resistance against the bacterial pathogen $P$. syringae.

NAI1 contributes to the defense response against $S$. exigua .

ER bodies have been proposed to participate in the defense response against herbivores (Nakano et al. 2014; Yamada et al. 2011). A recent study demonstrated increased susceptibility of 
the double mutant bglu18 pyk10 to the terrestrial isopod Armadillidium vulgare (Nakazaki et al. 2019b). The pyk10/bglu21 mutant forms ER bodies, although they are larger and less numerous than those in wild-type plants (Nagano et al. 2009). We measured the performance of the generalist leaf-chewing herbivore $S$. exigua in different ER body-related mutants. Performance assays have been widely used to study the defense mechanisms against herbivory (Chung et al. 2008; Cipollini et al. 2004; Müller et al. 2010;
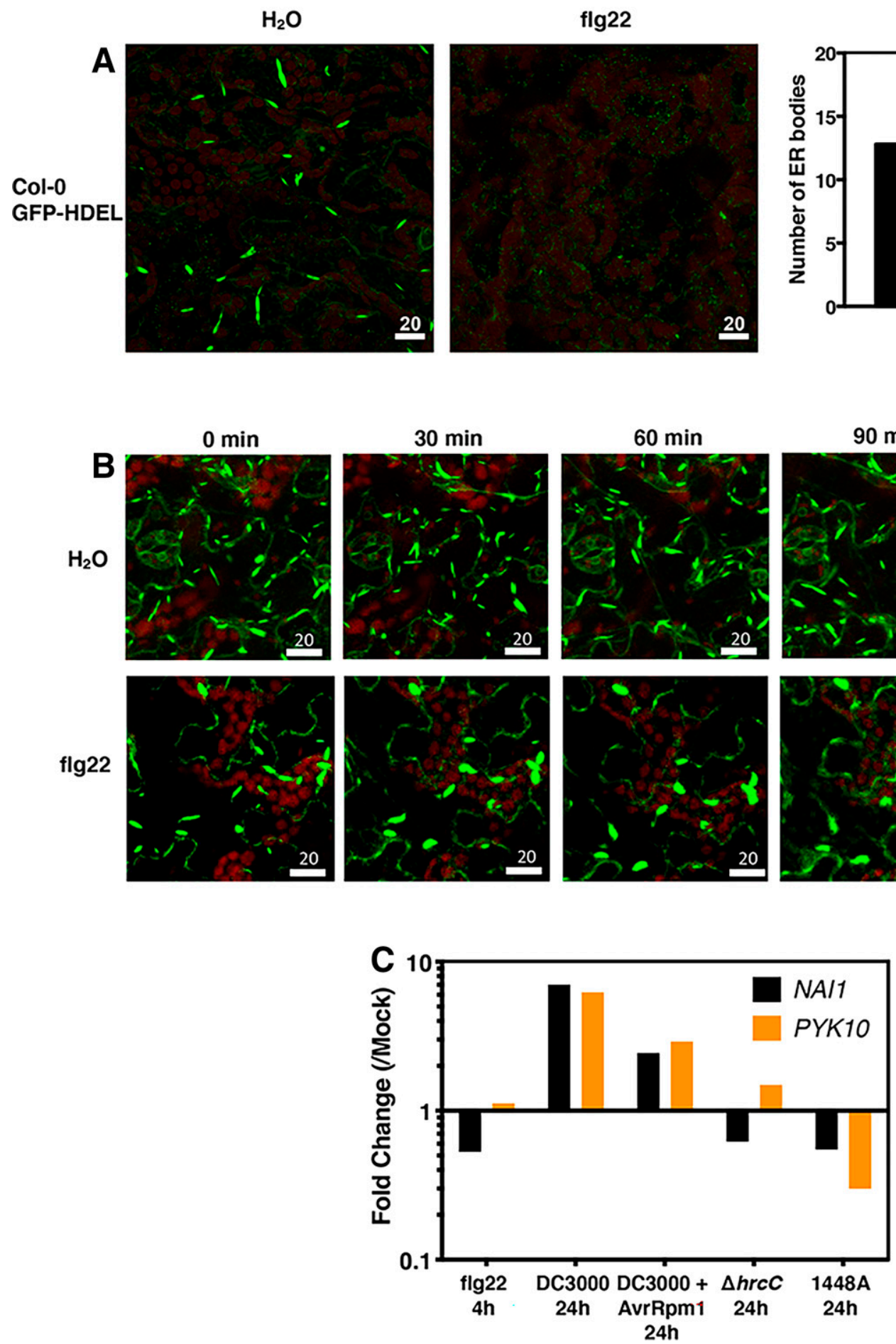

Fig. 1. Endoplasmic reticulum (ER) bodies are downregulated after activation of pattern-triggered immunity. A, Constitutive ER bodies disappear after flg22 treatment. Two-week-old Arabidopsis plants expressing green fluorescent protein with the ER-retention signal HDEL (GFP-HDEL) were vacuum-infiltrated with water or $100 \mathrm{nM}$ flg22. Cotyledons were observed in a Zeiss LSM710 confocal microscope $3 \mathrm{~h}$ after treatment. Red autofluorescence corresponds to chloroplasts. For ER body quantification, three fields from three independent cotyledons were used. ER bodies were quantified using Fiji. The experiment was repeated four times with similar results and a mean value \pm standard error of all quantifications is shown. Statistical differences were determined with a two-tailed $t$ test comparing water treatment with flg22. Three asterisks indicate $P<0.001$. Scale bar $=20 \mu \mathrm{m}$. B, Two-week-old Arabidopsis GFP-HDEL plants were vacuum infiltrated with water or $100 \mathrm{nM}$ flg22. The infiltrated cotyledons were monitored for 120 min, taking photos every 5 min with a Leica SP8 confocal microscope. C, Expression patterns of nail and pyk10 genes under different biotic stress. Data were obtained from the available microarray in eFP Browser. Five-week-old Col-0 plant leaves were syringe-infiltrated with either a 1- $\mu \mathrm{M}$ flg22 solution (using water as mock treatment) or the indicated bacterial strain at $10^{8} \mathrm{CFU}$ per milliliter (using $10 \mathrm{mM} \mathrm{MgCl}_{2}$ as mock treatment). Samples were taken $24 \mathrm{~h}$ after treatment. Fold change relative to mock treatment is shown. 

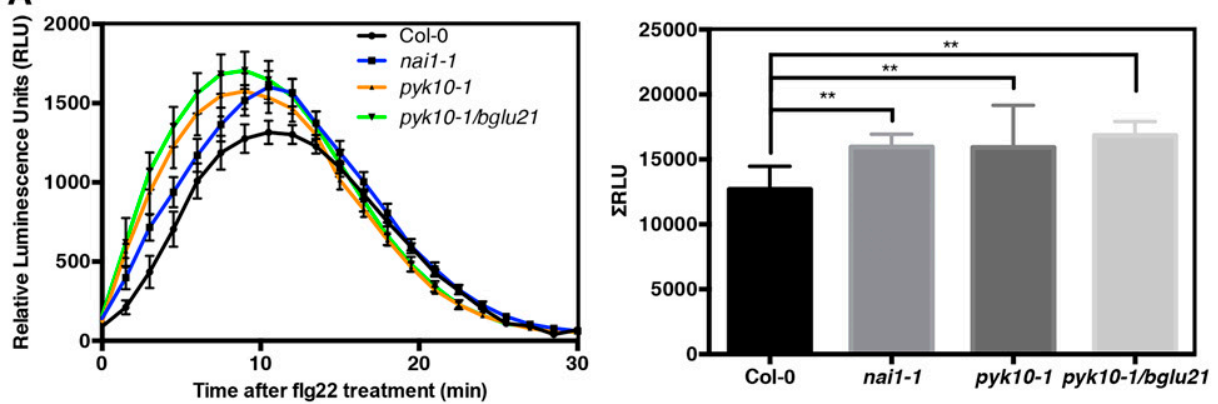

B
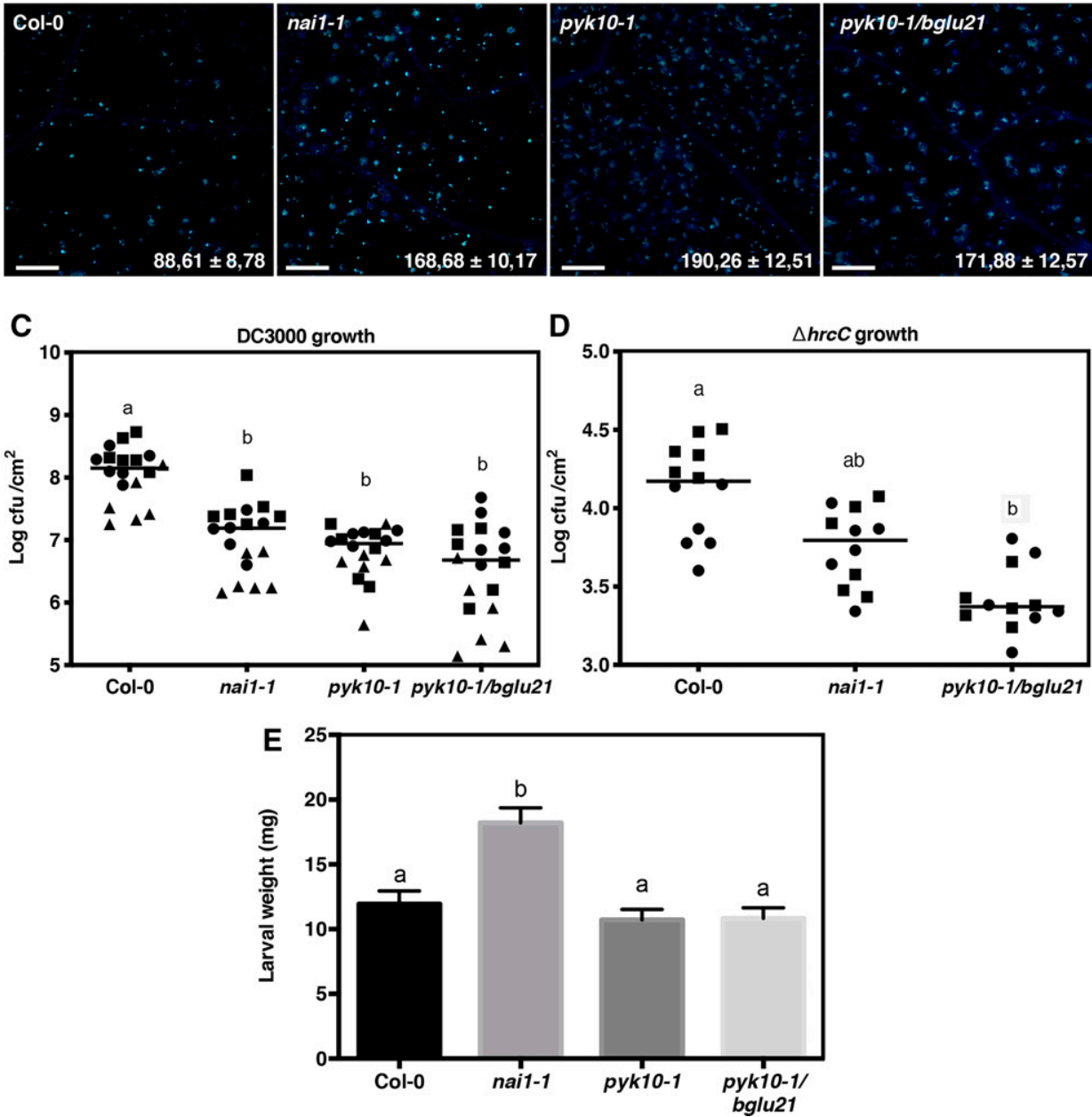

Fig. 2. Endoplasmic reticulum body mutants exhibit enhanced immune responses and resistance against Pseudomonas syringae. A, flg22-induced reactive oxygen species (ROS) burst in Col-0, nai1-1, pyk10-1, and pyk10-1/bglu21. Three-week-old Arabidopsis leaf discs were treated with $100 \mathrm{nM}$ flg22, and ROS were quantified using a luminescence based assay. The left graph represents the dynamics of the ROS produced in the different genotypes and the right graph represents the total relative light units (RLU) detected over a 30-min period. Error bars indicate standard deviation, $n=16$. Statistically significant differences were determined with one-way analysis of variance (ANOVA) $(\alpha=0.05)$ with Tukey's multiple comparisons test, and asterisks indicate statistical significance. The experiment was repeated four times with similar results and a representative experiment is shown. B, Quantification of flg22-induced callose deposition in Col-0, nai1-1, pyk10-1, and pyk10-1/bglu21. Four-week-old plants were infiltrated with $10 \mu \mathrm{M}$ flg22, and callose deposits were visualized under an epifluorescence microscope $18 \mathrm{~h}$ after treatment. Four images were taken per leaf and nine leaves were used per experiment. The mean number of deposits was quantified and is included \pm standard error (SE) in the bottom right corner of each representative image. The experiment was repeated three times with similar results, and a representative experiment is shown. White bar $=100 \mu \mathrm{m}$. C, Growth of P. syringae pv. tomato DC3000 in Col-0, nail-1, pyk10-1, and pyk10-1/bglu21. Five-week-old plant leaves were syringe-inoculated with a suspension of $5 \times 10^{4} \mathrm{CFU} / \mathrm{ml}$. Four days after inoculation, bacteria were recovered and quantified. Values from three independent replicates are shown $(n=18)$. Different symbols represent individual values from different replicates. Statistical differences were determined by ANOVA $(\alpha=0.05)$ with Tukey's multiple comparisons test, and different letters indicate statistical significance. D, Growth of the $P$. syringae pv. tomato $\Delta h r c C$ derivative in Col-0, nail-1, and pyk10-1/bglu21. Five-week-old plant leaves were syringe-inoculated with a suspension of $5 \times 10^{4} \mathrm{CFU} / \mathrm{ml}$. Four days after inoculation, bacteria were recovered and quantified. Values from three independent replicates are shown $(n=18)$. Different symbols represent individual values from different replicates. Statistical differences were determined by ANOVA $(\alpha=0.05)$ with Tukey's multiple comparisons test and different letters indicate statistical significance. E, Three-week-old Arabidopsis Col-0, nai1-1, pyk10-1, and pyk10-1/bglu21 plants were challenged with 3-day-old Spodoptera exigua larvae. Seven days after feeding, fresh weight of each larva was measured. Bars represent the mean weight \pm SE $(n=20)$. Statistically significant differences were determined with one-way ANOVA $(\alpha=0.05)$ with Tukey's multiple comparisons test and different letters indicate statistical significance. The experiment was repeated three times with similar results, and a representative experiment is shown. 
Santamaría et al. 2017; Van Oosten et al. 2008). We fed S. exigua larvae with Col-0 and the ER body mutants nail-1, pyk10-1, and pyk10-1/bglu21 plants and measured the weight of the larvae after 7 days. The fresh weight of $S$. exigua larvae was higher in nail-1 plants compared with the Col-0 control, confirming a role of ER bodies in the defense response against herbivore insects. Surprisingly, the performance of S. exigua in either pyk10-1 or pyk10-1/ bglu21 plants was not different compared Col-0, suggesting a redundant role of $P Y K 10$ and BGLU21 with other myrosinases in this context. (Fig. 2E).

\section{Coronatine induces the formation of ER bodies in rosette leaves.}

Transcriptomic analyses indicate that inoculation with virulent $P$. syringae pv. tomato DC3000 induces the expression of NAII and PYK10 (Fig. 1C). To check if this increased expression results in de novo formation of ER bodies, we inoculated rosette leaves of Arabidopsis GFP-HDEL plants with a relatively high inoculum (5 $\times 10^{7} \mathrm{CFU} / \mathrm{ml}$ ) of $P$. syringae pv. tomato DC3000, the derivative $\Delta h r c C$, and a coronatine mutant ( $\mathrm{cor}^{-}, P$. syringae pv. tomato DC3118). We applied the bacterial suspension using a brush to avoid wounding tissue. Three days after inoculation, we observed the samples under the confocal microscope (Fig. 3A). No ER bodies were found in the mock-treated leaves, in agreement with the absence of ER bodies in certain rosette leaves (Matsushima et al. 2002; Nakazaki et al. 2019b). Interestingly, a large number of ER bodies was found in leaves inoculated with wild-type $P$. syringae pv. tomato DC3000. The distribution of the ER bodies was patchy, suggesting that only some cells were forming the structure. To investigate if the formation of ER bodies localized surrounding bacterial colonies, we generated a $P$. syringae pv. tomato DC3000 derivative tagged with enhanced yellow fluorescent protein (eYFP). Three days after inoculation, we observed a large number of ER bodies surrounding the bacterial microcolony in Col-0 (Fig. 3B and C) but only a few aberrant ER bodies in the nail-1 mutant (Fig. 3C), suggesting that ER bodies induced by $P$. syringae pv. tomato DC3000 are dependent on NAII. To determine which bacterial factors are inducing the formation of ER bodies in rosette leaves, we inoculated Col-0 GFP-HDEL leaves with the $\Delta h r c C$ derivative and the cor $^{-}$mutant, both tagged with eYFP. We were unable to detect $\Delta h r c C$-eYFP microcolonies due to their extremely small size, consistent with their reduced growth in planta. Nevertheless, we did not detect ER bodies in leaves inoculated with the $\Delta h r c C$ mutant (Fig. 3A). Interestingly, the cor $^{-}$eYFP mutant was unable to induce the formation of de novo ER bodies, consistent with the role of coronatine mimicking JA (Fig. 3). This result indicates that induction of ER bodies could be a virulence function of coronatine. Analyzing transcriptomic data of plants inoculated either with $P$. syringae pv. tomato DC3000 or the cor $^{-}$mutant, we observed that many genes related to ER bodies, including NAI1, PYK10, and BGLU18, were upregulated by $P$. syringae pv. tomato DC3000 but not after inoculation with $P$. syringae pv. tomato DC3118 ( cor $^{-}$) (Supplementary Fig. S3) (Thilmony et al. 2006). To investigate the ability of coronatine to promote bacterial virulence in the absence of wild-type ER body formation, we measured the growth of $P$. syringae pv. tomato DC3000 and the $P$. syringae pv. tomato cor ${ }^{-}$mutant in Col-0, nail-1, pyk10-1, and pyk10-1/bglu21. In order to bypass the role of coronatine on reopening stomata (Melotto et al. 2006, 2008), we performed syringe inoculation in 5-week-old plant leaves. Four days after inoculation, a strong growth restriction was observed in the coronatine mutant compared with wildtype $P$. syringae pv. tomato DC3000 in Col-0 (Fig. 3D). In the ER body-defective backgrounds, a stronger growth restriction was observed for P. syringae pv. tomato DC3000 (Figs. 2C and $3 \mathrm{D})$. However, the growth of the coronatine mutant was not significantly different between wild-type Col-0 and the ER body mutants.

\section{DISCUSSION}

The main contents in ER bodies are $\beta$-glucosidases, enzymes that can produce toxic compounds to protect the plant from herbivore attack (Matsushima et al. 2003). Induction of ER bodies by wounding or by MeJA treatment suggests an association with plant defense (Nakano et al. 2014). However, there is little experimental evidence of the association between ER bodies and plant immunity. Plants are infected by diverse pathogens that often utilize opposing virulence strategies and are differentially impacted by plant immune responses (e.g., chewing insects versus biotrophic or hemibiotrophic pathogens). In this study we demonstrate contrasting roles of ER bodies in the plant response to pathogenic bacteria and herbivores.

Previous studies have implicated ER bodies in plant protection against fungi and feeding insects. Sherameti et al. (2008) demonstrated that $P Y K 10$ is required to protect plants against excessive root colonization by the endophytic fungus Piriformospora indica. Furthermore, Nakazaki et al. (2019b) showed that bglul8 pyk10 double mutant plants are more susceptible than wild type to the attack of the terrestrial isopod Armadillidium vulgare (pillbug). In this work, we experimentally link the function of the ER bodies with the plant response against the bacterial pathogen $P$. syringae and the herbivore $S$. exigua. We found an increased weight acquisition of the generalist chewing herbivore $S$. exigua in nail-1 plants, compared with Col-0, but not in pyk10-1 and pyk10-1/ bglu21 mutants (Fig. 2E). S. exigua feeds on leaves, in which constitutive ER bodies are absent (Matsushima et al. 2002). Constitutive leaf ER bodies, which are dependent on NAIl, localize in the edges of the leaves and mainly contain PYK10 and BGLU18 (Nakazaki et al. 2019b). The biogenesis and content of leaf ER bodies is regulated by both JA-dependent and -independent pathways (Nakazaki et al. 2019a). While NAIl expression and, therefore, PYK10 accumulation is dependent on JA, BGLU18 accumulation in leaf ER bodies is independent of JA (Nakazaki et al. 2019a). Since performance of S. exigua is not affected in pyk10-1 mutants, the genetic analysis points to BGLU18 as responsible for the defense response against $S$. exigua. Furthermore, chewing induces JA accumulation (Rehrig et al. 2014), and this signal would potentially lead to the formation of the inducible ER bodies. The nail mutant is compromised in formation of inducible local and systemic ER bodies. In the nail mutant, inducible ER bodies are long and tubular and mainly observed in cells surrounding the wound site (Ogasawara et al. 2009). We also observed that $P$. syringae pv. tomato DC3000 was unable to induce ER body formation in the nail mutant, indicating that the absence or altered composition or structure of ER bodies in NAIl suppresses growth of the hemibiotrophic pathogen $P$. syringae, while enhancing performance of a chewing herbivore.

$P$. syringae pv. tomato DC3000 induces the formation of ER bodies in leaves. Since the flagellin peptide flg22 downregulates the formation of constitutive ER bodies and we have shown that ER bodies negatively regulate PTI, we hypothesize that $P$. syringae induction of ER bodies is a virulence strategy to suppress host immune responses. The two main virulence factors of $P$. syringae pv. tomato are the phytotoxin coronatine and the T3SS effectors. The bacterial phytotoxin coronatine, which mimics the function of JA in the plant (Bender et al. 1999), induces the formation of ER bodies in leaves (Fig. 3A and B). JA is perceived in the plant through degradation of JASMONATE ZIM-DOMAIN (JAZ) transcriptional repressors and JAZ proteins suppress the expression of ER body components as well as indole glucosinolates (Ghorbel et al. 2021; Guo et al. 2018). Thus, JA or pathogen-JA mimics can regulate the abundance of this specialized organelle 

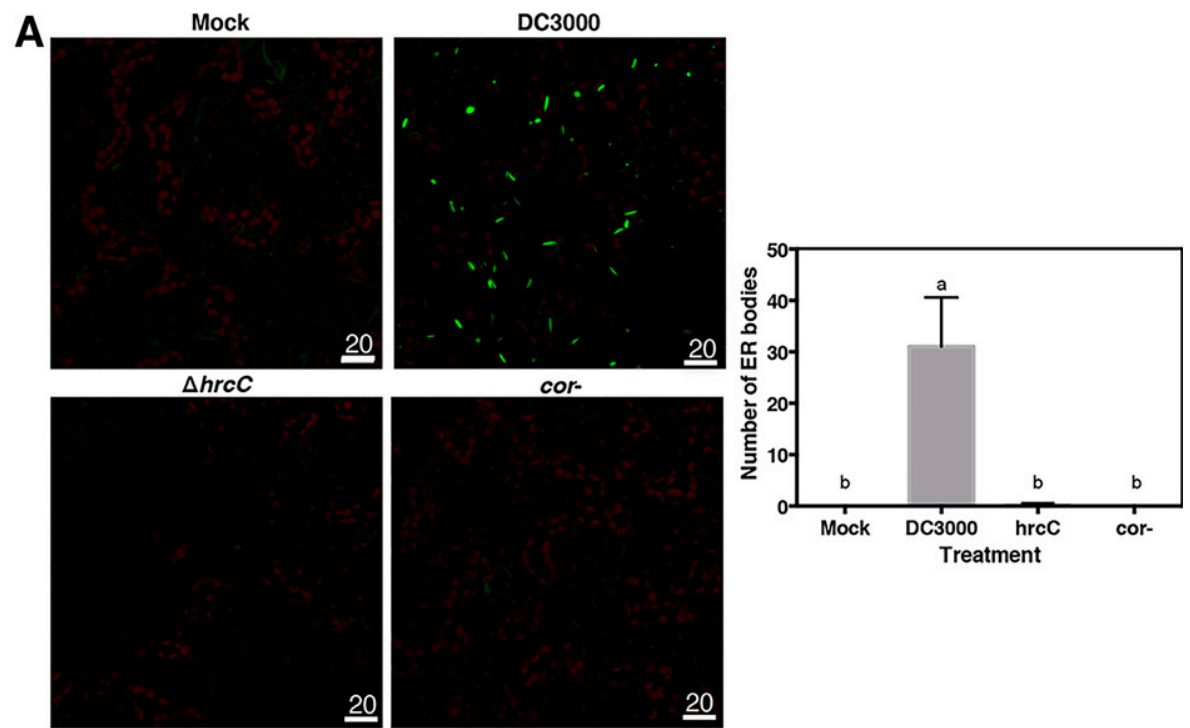

B DC3000 eYFP

cor - eYFP
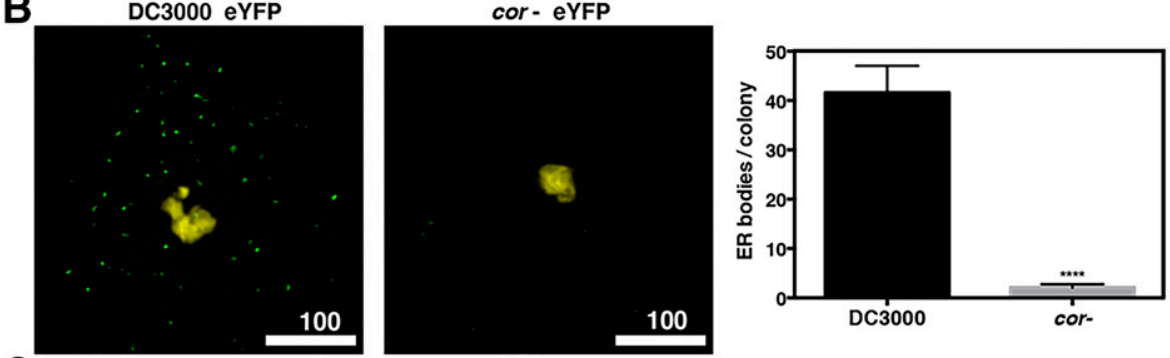

C Col-0 GFP-HDEL nai1-1 GFP-HDEL
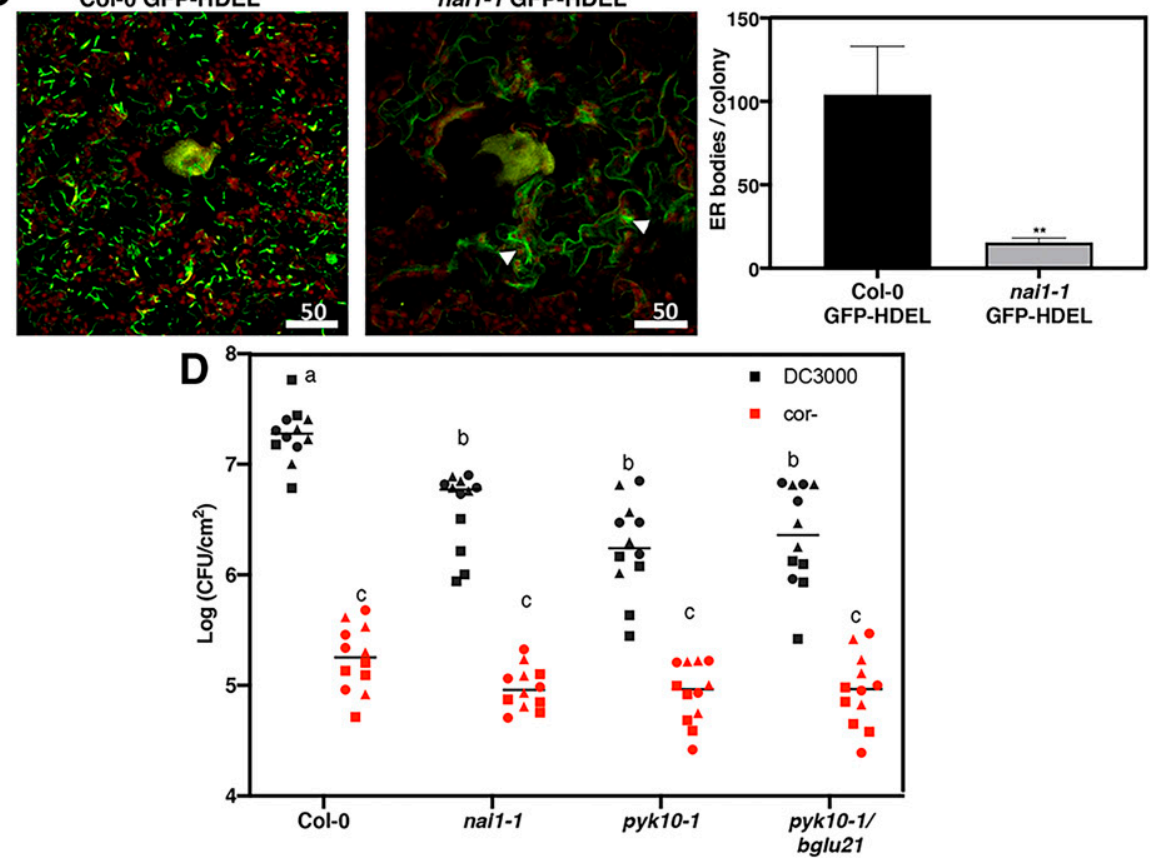

Fig. 3. Coronatine induces formation of endoplasmic reticulum (ER) bodies in leaves and its virulence function is compromised in mutants impaired in ER body formation. A to C, Confocal microscope images of bacterial microcolonies (yellow) and ER bodies (green). Five-week-old Arabidopsis plants expressing green fluorescent protein with the ER-retention signal HDEL (GFP-HDEL) were brush-inoculated with a suspension containing $5 \times 10^{7} \mathrm{CFU} / \mathrm{ml}(\mathrm{A})$ or syringe inoculated with a bacterial suspension containing $5 \times 10^{4} \mathrm{CFU} / \mathrm{ml}$ (B and $\mathrm{C}$ ). Three days after inoculation, leaf sections were visualized under the confocal microscope. A representative $z$-stack projection image is shown. ER bodies were quantified from the green channel using Fiji. Bars represent the mean number of ER bodies (A) or the number of ER bodies surrounding a single bacterial micro colony (B, C) \pm standard error. Red autofluorescence corresponds to chloroplasts. The experiments were repeated three times with similar results, and the mean of all experiments is shown. Statistically significant differences were determined with one-way analysis of variance (ANOVA) $(\alpha=0.05)$ with Tukey's multiple comparison test (A) and different letters indicate statistical significance. Data in B and C were analyzed by $t$ test $(\alpha=0.001)$. White bar $=20 \mu \mathrm{m}(\mathrm{A}), 100 \mu \mathrm{m}(\mathrm{B}), 50 \mu \mathrm{m}(\mathrm{C})$. D, Growth of Pseudomonas syringae pv. tomato DC3000 and its derivative coronatine mutant (cor-, DC3118) in Col-0, nail-1, pyk10-1, and pyk10-1/bglu21. Five-week-old plant leaves were syringe-inoculated with a bacterial suspension of $5 \times 10^{4} \mathrm{CFU} / \mathrm{ml}$. Four days after inoculation, bacteria were recovered and quantified. Values from three independent replicates are shown $(n=12)$. Different symbols represent individual values from different replicates. Statistically significant differences were determined by ANOVA $(\alpha=0.05)$ with Tukey's multiple comparisons test and different letters indicate statistical significance. 
to induce or suppress defense, depending on pathogen type. The most studied coronatine virulence function is to reopen stomata upon PAMP-induced closure (Melotto et al. 2006, 2008). However, coronatine also has been shown to suppress immunity once the bacteria colonize the apoplast (Fig. 3C) (Geng et al. 2012). The T3SS defective mutant $\Delta h r c C$ is not able to induce the formation of ER bodies, which would indicate an effect of T3Es on the induction of ER bodies. However, we cannot rule out that the absence of ER bodies is due to an insufficient amount of coronatine produced by the $\Delta h r c C$ microcolonies.

Bacterial flg22 perception leads to a downregulation of the ER bodies at both transcriptional and posttranscriptional levels (Fig. 1; Supplementary Fig. S1). We found that Arabidopsis mutants defective in either ER body biogenesis or composition are more resistant to pathogenic bacteria (Fig. 2; Supplementary Fig. S2). The main components of ER bodies are myrosinases, enzymes that modify glucosinolates to initiate the glucosinolate breakdown, which will result in different products depending on the biochemical properties of the modifier protein and the chemical nature of the glucosinolate side chain (Sugiyama and Hirai 2019; Wittstock and Burow 2010). Those products can function as defense compounds or as signaling molecules.

PYK10/BGLU23 is an atypical myrosinase similar to PENETRATION 2 (PEN2, BGLU26) that hydrolyzes indole glucosinolates (IGs) (Nakano et al. 2017; Nakazaki et al. 2019b). PEN2-mediated conversion of $4 \mathrm{MI} 3 \mathrm{G}$ into glucosinolatederived products plays an important role in the defense response against fungi (Bednarek et al. 2009). Interestingly, PEN2 is required for callose deposition upon flg22 perception and for resistance against $P$. syringae pv. tomato DC3000 infection in seedlings grown in liquid media (Clay et al. 2009; Johansson et al. 2014). In contrast, in adult plants grown in soil, callose deposition and $P$. syringae pv. tomato DC3000 growth is not affected in the pen2-1 mutant (Geng et al. 2012). The pen2-1 mutant grown in soil exhibits enhanced resistance to $P$. syringae $\mathrm{pv}$. maculicola (Stahl et al. 2016). These apparently contradictory results indicate that PEN2 activity is dependent on one or both the age and the metabolic state of the plant. $4 \mathrm{MI} 3 \mathrm{G}$ overaccumulates in homogenized tissue of bglu18/pyk10 mutants indicating that one or both of these $\beta$-glucosidases hydrolyze $4 \mathrm{MI} 3 \mathrm{G}$ (Nakazaki et al. 2019b). Our results indicate that the single pyk10-1 mutant and the double pyk10-1/bglu21 mutant accumulate an increased number of callose deposits in response to flg22 treatment and are more resistant to $P$. syringae pv. tomato DC3000. Although both PEN2 and PYK10 seem to have the same substrate (4MI3G), their respective mutants differ on phenotypes such as flg22-induced callose deposition and resistance to P. syringae pv. tomato DC3000 (Clay et al. 2009; Johansson et al. 2014). Apart from the above-mentioned effect related to growth conditions, one of the reasons explaining these differences could be the different compartmentalization of both PEN2 and PYK10. While PEN2 is localized in peroxisome and mitochondria (Fuchs et al. 2016; Lipka et al. 2005), PYK10 localizes in ER bodies (Matsushima et al. 2003). Furthermore, myrosinase activity is regulated by different myrosinasebiding proteins and myrosinase-associated proteins as well as different specifier proteins, which lead to the formation of different breakdown products (Wittstock et al. 2016). This variety of compounds can have a direct or indirect effect on the plant defense response (Wittstock et al. 2016). It is possible that mutants lacking ER bodies or core components of ER bodies also exhibit heightened basal defense, resulting in enhanced ROS and callose production.

In conclusion, we have demonstrated the involvement of ER body-dependent regulation of immunity to bacteria and induction of ER bodies as a pathogen virulence strategy. Further work will shed light on how this novel function of ER bodies influences bacterial-plant interactions and pave the way for the discovery of the role of indole-glucosinolates breakdown on the response against bacterial invasion in plants.

\section{MATERIALS AND METHODS}

\section{Plant material and growth conditions.}

A. thaliana Col-0, the transgenic line expressing GFP-HDEL (Hayashi et al. 2001), and the mutants nail-1 (Matsushima et al. 2003), pyk10-1 (Nagano et al. 2008), pyk10-1/bglu21 (Nagano et al. 2009), bglu18-1 (Ogasawara et al. 2009), and pbpl-1 (Nagano et al. 2005) were grown in a controlled environment chamber at $23^{\circ} \mathrm{C}, 70 \%$ relative humidity, and a 10 -h light and 14-h dark photoperiod with light intensity of $100 \mu \mathrm{E} \mathrm{m}^{-2} \cdot \mathrm{s}^{-1}$. All seeds were stratified for 3 to 4 days at $4^{\circ} \mathrm{C}$ before sowing into soil.

\section{Bacterial strains, growth conditions, and pathogen assays.}

Pseudomonas syringae pv. tomato DC3000 (Cuppels 1986) and the derivative strains $\Delta h r c C$ and $\operatorname{cor}^{-}\left(\mathrm{cma}^{-} \mathrm{cfa}^{-}\right.$[Brooks et al. 2004]) were grown at $28^{\circ} \mathrm{C}$ in lysogeny broth medium (Bertani 1951). Antibiotics were used when appropriate, at the following concentrations: rifampicin, $100 \mu \mathrm{g} / \mathrm{ml}$ ); kanamycin, $25 \mu \mathrm{g} / \mathrm{ml}$; and gentamycin, $10 \mu \mathrm{g} / \mathrm{ml}$.

The $P$. syringae pv. tomato DC3000, $\Delta h r c C$, and cor $^{-}$eYFP derivatives were generated using a Tn7 delivery system as previously described (Rufián et al. 2018)

For pathogen assays, 5-week-old Arabidopsis plants were inoculated with a $P$. syringae suspension of $5 \times 10^{4} \mathrm{CFU} / \mathrm{ml}$ prepared in $10 \mathrm{mM} \mathrm{MgCl}_{2}$. Three leaves per plant were syringe-infiltrated and five plants were used for each experiment. Four days after inoculation, three leaf discs of $1 \mathrm{~cm}$ diameter (one per leaf) were ground in $1 \mathrm{ml}$ of $10 \mathrm{mM} \mathrm{MgCl}$, and serial dilutions were plated.

\section{Transcriptomic analyses.}

For the analysis of NAI1 and PYK10 expression under different biotic stresses, we used transcriptomic data available in eFP Browser. Specifically, we used the dataset AtGenExpress: response to virulent, avirulent, T3SS-deficient, and nonhost bacteria (NASCArrays-120). Five-week-old Col-0 plants were infiltrated with the corresponding $P$. syringae strain. Samples were taken $24 \mathrm{~h}$ after treatment, and RNA was isolated and hybridized to the ATH1 GeneChip. The data were normalized by GCOS normalization, $\mathrm{TGT}=100$.

\section{flg22-related assays.}

For ROS burst assays, leaf discs were collected, using a cork borer of $5 \mathrm{~mm}$ diameter from 3-week-old Arabidopsis plants, and were floated overnight in deionized water. The next day, the water was replaced with an assay solution containing $17 \mathrm{mg}$ of luminol per milliliter (Sigma), $10 \mathrm{mg}$ of horseradish peroxidase per milliliter (Sigma), and $100 \mathrm{nM}$ flg22 (Genscript). Luminescence was measured using Tristar multimode reader (Berthold Technology).

Callose deposits were quantified from 4-week-old Arabidopsis leaves infiltrated with a $10 \mu \mathrm{M}$ flg22 solution. Eighteen hours after treatment, leaves were detached and incubated 15 $\min$ at $65^{\circ} \mathrm{C}$ in $5 \mathrm{ml}$ of alcoholic lactophenol (one volume of phenol/glycerol/lactic acid/water [1:1:1:1] and two volumes of ethanol). Leaves were transferred to fresh alcoholic lactophenol and were incubated overnight at room temperature. After two washes with $50 \%$ ethanol, leaves were stained with aniline blue and callose deposits were visualized under a fluorescence microscope. Quantification of callose deposits was performed using the Fiji distribution of ImageJ. 


\section{Confocal microscopy.}

Two-week old Arabidopsis GFP-HDEL plants were vacuuminfiltrated with either water or $100 \mathrm{nM}$ flg22. Three hours after the treatment, cotyledons were detached and observed under a Zeiss LSM710 confocal microscope equipped with an LDC-apochromat $40 \times / 1.1 \mathrm{~W}$ Korr M27 water-immersion objective (NA 1.1). GFP was excited at $488 \mathrm{~nm}$ and emission was collected at 500 to $550 \mathrm{~nm}$. For monitoring of the dynamics of the ER bodies, cotyledons were treated as above and were visualized using a Leica SP8 confocal microscope (Leica Microsystems $\mathrm{GmbH}$ ). GFP was excited at $488 \mathrm{~nm}$ and emission collected at 500 to $550 \mathrm{~nm}$.

For visualization of bacterial-induced ER bodies, 5-week old Arabidopsis GFP-HDEL plants were brush-inoculated with a 5 $\times 10^{7} \mathrm{CFU} / \mathrm{ml}$ suspension of the indicated strain (Fig. 3A). Three days after inoculation, leaves were observed as described above.

For visualization of bacterial microcolonies, 5-week old Arabidopsis GFP-HDEL plants were syringe-infiltrated with a $5 \times 10^{4}$ $\mathrm{CFU} / \mathrm{ml}$ suspension and leaves were visualized using the Leica SP5 II confocal microscope (Leica Microsystems GmbH). Images of eYFP and GFP were sequentially obtained using the following conditions (excitation/emission): eYFP (514/525 to $600 \mathrm{~nm}$ ), GFP (488/500 to $525 \mathrm{~nm}$ ). Z series imaging was taken at $1 \mu \mathrm{m}$ interval. Image processing was performed using Leica LAS AF (Leica Microsystems). ER bodies were quantified using Fiji distribution of ImageJ software.

\section{S. exigua performance.}

S. exigua eggs were provided by S. Herrero (University of Valencia). After eclosion, S. exigua larvae were fed with an artificial diet (Elvira et al. 2010). Three days after eclosion, five larvae of approximately the same size were transferred to a 4- to 5-weekold Arabidopsis plant grown in Jiffy-7 pods. Larvae were transferred to new plants every $24 \mathrm{~h}$ to avoid food privation. Seven days after plant feeding, fresh weight of the larvae was measured. For each experiment, five replicates were performed (25 larvae per genotype).

\section{ACKNOWLEDGMENTS}

We thank A. Macho for hosting J. S. Rufián in his research laboratory and providing feedback on the manuscript. We thank D. Kliebenstein for evaluating the manuscript and providing feedback.

\section{LITERATURE CITED}

Bednarek, P., Piślewska-Bednarek, M., Svatos, A., Schneider, B., Doubsky, J., Mansurova, M., Humphry, M., Consonni, C., Panstruga, R., SanchezVallet, A., Molina, A., and Schulze-Lefert, P. 2009. A glucosinolate metabolism pathway in living plant cells mediates broad-spectrum antifungal defense. Science 323:101-106.

Bender, C. L., Alarcón-Chaidez, F., and Gross, D. C. 1999. Pseudomonas syringae phytotoxins: Mode of action, regulation, and biosynthesis by peptide and polyketide synthetases. Microbiol. Mol. Biol. Rev. 63: 266-292.

Bertani, G. 1951. Studies on lysogenesis. I. The mode of phage liberation by lysogenic Escherichia coli. J. Bacteriol. 62:293-300.

Bjornson, M., Pimprikar, P., Nürnberger, T., and Zipfel, C. 2021. The transcriptional landscape of Arabidopsis thaliana pattern-triggered immunity. Nat. Plants 7:579-586.

Brooks, D. M., Hernández-Guzmán, G., Kloek, A. P., Alarcón-Chaidez, F., Sreedharan, A., Rangaswamy, V., Peñaloza-Vázquez, A., Bender, C. L., and Kunkel, B. N. 2004. Identification and characterization of a well-defined series of coronatine biosynthetic mutants of Pseudomonas syringae pv. tomato DC3000. Mol. Plant-Microbe Interact. 17: $162-174$.

Chung, H. S., Koo, A. J. K., Gao, X., Jayanty, S., Thines, B., Jones, A. D., and Howe, G. A. 2008. Regulation and function of Arabidopsis JASMONATE ZIM-domain genes in response to wounding and herbivory. Plant Physiol. 146:952-964.
Cipollini, D., Enright, S., Traw, M. B., and Bergelson, J. 2004. Salicylic acid inhibits jasmonic acid-induced resistance of Arabidopsis thaliana to Spodoptera exigua. Mol. Ecol. 13:1643-1653.

Clay, N. K., Adio, A. M., Denoux, C., Jander, G., and Ausubel, F. M. 2009. Glucosinolate metabolites required for an Arabidopsis innate immune response. Science 323:95-101.

Couto, D., and Zipfel, C. 2016. Regulation of pattern recognition receptor signalling in plants. Nat. Rev. Immunol. 16:537-552.

Cuppels, D. A. 1986. Generation and characterization of $\mathrm{Tn} 5$ insertion mutations in Pseudomonas syringae pv. tomato. Appl. Environ. Microbiol. 51:323-327.

Elvira, S., Gorría, N., Muñoz, D., Williams, T., and Caballero, P. 2010. A simplified low-cost diet for rearing Spodoptera exigua (Lepidoptera: Noctuidae) and its effect on S. exigua nucleopolyhedrovirus production. J. Econ. Entomol. 103:17-24.

Fuchs, R., Kopischke, M., Klapprodt, C., Hause, G., Meyer, A. J., Schwarzländer, M., Fricker, M. D., and Lipka, V. 2016. Immobilized subpopulations of leaf epidermal mitochondria mediate PENETRATION2-dependent pathogen entry control in Arabidopsis. Plant Cell 28:130-145.

Geng, X., Cheng, J., Gangadharan, A., and Mackey, D. 2012. The coronatine toxin of Pseudomonas syringae is a multifunctional suppressor of Arabidopsis defense. Plant Cell 24:4763-4774.

Ghorbel, M., Brini, F., Sharma, A., and Landi, M. 2021. Role of jasmonic acid in plants: The molecular point of view. Plant Cell Rep. 10.1007/ s00299-021-02687-4. Published online.

Gimenez-Ibanez, S., Boter, M., Ortigosa, A., García-Casado, G., Chini, A., Lewsey, M. G., Ecker, J. R., Ntoukakis, V., and Solano, R. 2017. JAZ2 controls stomata dynamics during bacterial invasion. New Phytol. 213: 1378-1392.

Gómez-Gómez, L., and Boller, T. 2000. FLS2: An LRR receptor-like kinase involved in the perception of the bacterial elicitor flagellin in Arabidopsis. Mol. Cell 5:1003-1011.

Guo, Q., Yoshida, Y., Major, I. T., Wang, K., Sugimoto, K., Kapali, G., Havko, N. E., Benning, C., and Howe, G. A. 2018. JAZ repressors of metabolic defense promote growth and reproductive fitness in Arabidopsis. Proc. Natl. Acad. Sci. U.S.A. 115:E10768-E10777.

Hayashi, Y., Yamada, K., Shimada, T., Matsushima, R., Nishizawa, N. K., and Hara-Nishimura, I. 2001. A proteinase-storing body that prepares for cell death or stresses in the epidermal cells of Arabidopsis. Plant Cell Physiol. 42:894-899.

Henry, E., Yadeta, K. A., and Coaker, G. 2013. Recognition of bacterial plant pathogens: Local, systemic and transgenerational immunity. New Phytol. 199:908-915.

Johansson, O. N., Fantozzi, E., Fahlberg, P., Nilsson, A. K., Buhot, N., Tör, M., and Andersson, M. X. 2014. Role of the penetrationresistance genes PEN1, PEN2 and PEN3 in the hypersensitive response and race-specific resistance in Arabidopsis thaliana. Plant J. 79:466-476.

Lahrmann, U., Strehmel, N., Langen, G., Frerigmann, H., Leson, L., Ding, Y., Scheel, D., Herklotz, S., Hilbert, M., and Zuccaro, A. 2015. Mutualistic root endophytism is not associated with the reduction of saprotrophic traits and requires a noncompromised plant innate immunity. New Phytol. 207:841-857.

Lipka, V., Dittgen, J., Bednarek, P., Bhat, R., Wiermer, M., Stein, M., Landtag, J., Brandt, W., Rosahl, S., Scheel, D., Llorente, F., Molina, A., Parker, J., Somerville, S., and Schulze-Lefert, P. 2005. Pre- and postinvasion defenses both contribute to nonhost resistance in Arabidopsis. Science 310:1180-1183.

Macho, A. P. 2016. Subversion of plant cellular functions by bacterial type-III effectors: Beyond suppression of immunity. New Phytol. 210:51-57.

Matsushima, R., Fukao, Y., Nishimura, M., and Hara-Nishimura, I. 2004. NAI1 gene encodes a basic-helix-loop-helix-type putative transcription factor that regulates the formation of an endoplasmic reticulumderived structure, the ER body. Plant Cell 16:1536-1549.

Matsushima, R., Hayashi, Y., Kondo, M., Shimada, T., Nishimura, M., and Hara-Nishimura, I. 2002. An endoplasmic reticulum-derived structure that is induced under stress conditions in Arabidopsis. Plant Physiol. 130:1807-1814.

Matsushima, R., Hayashi, Y., Yamada, K., Shimada, T., Nishimura, M., and Hara-Nishimura, I. 2003. The ER body, a novel endoplasmic reticulumderived structure in Arabidopsis. Plant Cell Physiol. 44:661-666.

Melotto, M., Underwood, W., and He, S. Y. 2008. Role of stomata in plant innate immunity and foliar bacterial diseases. Annu. Rev. Phytopathol. 46:101-122.

Melotto, M., Underwood, W., Koczan, J., Nomura, K., and He, S. Y. 2006. Plant stomata function in innate immunity against bacterial invasion. Cell 126:969-980. 
Millet, Y. A., Danna, C. H., Clay, N. K., Songnuan, W., Simon, M. D., Werck-Reichhart, D., and Ausubel, F. M. 2010. Innate immune responses activated in Arabidopsis roots by microbe-associated molecular patterns. Plant Cell 22:973-990.

Mitsuhashi, N., Shimada, T., Mano, S., Nishimura, M., and Hara-Nishimura, I. 2000. Characterization of organelles in the vacuolar-sorting pathway by visualization with GFP in tobacco BY-2 cells. Plant Cell Physiol. 41:9931001.

Müller, R., de Vos, M., Sun, J. Y., Sønderby, I. E., Halkier, B. A., Wittstock, U., and Jander, G. 2010. Differential effects of indole and aliphatic glucosinolates on lepidopteran herbivores. J. Chem. Ecol. 36:905 913.

Nagano, A. J., Fukao, Y., Fujiwara, M., Nishimura, M., and Hara-Nishimura, I. 2008. Antagonistic jacalin-related lectins regulate the size of ER body-type beta-glucosidase complexes in Arabidopsis thaliana. Plant Cell Physiol. 49:969-980.

Nagano, A. J., Maekawa, A., Nakano, R. T., Miyahara, M., Higaki, T., Kutsuna, N., Hasezawa, S., and Hara-Nishimura, I. 2009. Quantitative analysis of ER body morphology in an Arabidopsis mutant. Plant Cell Physiol. 50:2015-2022.

Nagano, A. J., Matsushima, R., and Hara-Nishimura, I. 2005. Activation of an ER-body-localized beta-glucosidase via a cytosolic binding partner in damaged tissues of Arabidopsis thaliana. Plant Cell Physiol. 46:11401148 .

Nakano, R. T., Piślewska-Bednarek, M., Yamada, K., Edger, P. P., Miyahara, M., Kondo, M., Böttcher, C., Mori, M., Nishimura, M., Schulze-Lefert, P., Hara-Nishimura, I., and Bednarek, P. 2017. PYK10 myrosinase reveals a functional coordination between endoplasmic reticulum bodies and glucosinolates in Arabidopsis thaliana. Plant J. 89:204220

Nakano, R. T., Yamada, K., Bednarek, P., Nishimura, M., and HaraNishimura, I. 2014. ER bodies in plants of the Brassicales order: Biogenesis and association with innate immunity. Front. Plant Sci. 5:73.

Nakazaki, A., Yamada, K., Kunieda, T., Sugiyama, R., Hirai, M. Y., Tamura, K., Hara-Nishimura, I., and Shimada, T. 2019b. Leaf endoplasmic reticulum bodies identified in Arabidopsis rosette leaves are involved in defense against herbivory. Plant Physiol. 179: $1515-1524$

Nakazaki, A., Yamada, K., Kunieda, T., Tamura, K., Hara-Nishimura, I., and Shimada, T. 2019a. Biogenesis of leaf endoplasmic reticulum body is regulated by both jasmonate-dependent and independent pathways. Plant Signal. Behav. 14:1622982.

Ogasawara, K., Yamada, K., Christeller, J. T., Kondo, M., Hatsugai, N., Hara-Nishimura, I., and Nishimura, M. 2009. Constitutive and inducible ER bodies of Arabidopsis thaliana accumulate distinct beta-glucosidases. Plant Cell Physiol. 50:480-488.

Rehrig, E. M., Appel, H. M., Jones, A. D., and Schultz, J. C. 2014. Roles for jasmonate- and ethylene-induced transcription factors in the ability of
Arabidopsis to respond differentially to damage caused by two insect herbivores. Front. Plant Sci. 5:407.

Rufián, J. S., Macho, A. P., Corry, D. S., Mansfield, J. W., Ruiz-Albert, J., Arnold, D. L., and Beuzón, C. R. 2018. Confocal microscopy reveals in planta dynamic interactions between pathogenic, avirulent and nonpathogenic Pseudomonas syringae strains. Mol. Plant Pathol. 19:537551.

Santamaría, M. E., Martinez, M., Arnaiz, A., Ortego, F., Grbic, V., and Diaz, I. 2017. MATI, a novel protein involved in the regulation of herbivore-associated signaling pathways. Front. Plant Sci. 8:975.

Sherameti, I., Venus, Y., Drzewiecki, C., Tripathi, S., Dan, V. M., Nitz, I. Varma, A., Grundler, F. M., and Oelmüller, R. 2008. PYK10, a betaglucosidase located in the endoplasmatic reticulum, is crucial for the beneficial interaction between Arabidopsis thaliana and the endophytic fungus Piriformospora indica. Plant J. 54:428-439.

Stahl, E., Bellwon, P., Huber, S., Schlaeppi, K., Bernsdorff, F., VallatMichel, A., Mauch, F., and Zeier, J. 2016. Regulatory and functional aspects of indolic metabolism in plant systemic acquired resistance. Mol. Plant. 9:662-681.

Sugiyama, R., and Hirai, M. Y. 2019. Atypical myrosinase as a mediator of glucosinolate functions in plants. Front. Plant Sci. 10:1008.

Thilmony, R., Underwood, W., and He, S. Y. 2006. Genome-wide transcriptional analysis of the Arabidopsis thaliana interaction with the plant pathogen Pseudomonas syringae pv. tomato DC3000 and the human pathogen Escherichia coli O157:H7. Plant J. 46:34-53.

Van Oosten, V. R., Bodenhausen, N., Reymond, P., Van Pelt, J. A., Van Loon, L. C., Dicke, M., and Pieterse, C. M. J. 2008. Differential effectiveness of microbially induced resistance against herbivorous insects in Arabidopsis. Mol. Plant-Microbe Interact. 21:919-930.

Weiler, E. W., Kutchan, T. M., Gorba, T., Brodschelm, W., Niesel, U., and Bublitz, F. 1994. The Pseudomonas phytotoxin coronatine mimics octadecanoid signalling molecules of higher plants. FEBS Lett. 345:9-13.

Wittstock, U., and Burow, M. 2010. Glucosinolate breakdown in Arabidopsis: Mechanism, regulation and biological significance. The Arabidopsis Book 8:e0134.

Wittstock, U., Kurzbach, E., Herfurth, A. M., and Stauber, E. J. 2016. Glucosinolate breakdown. Adv. Bot. Res. 80:125-169.

Xu, Z., Escamilla-Treviño, L., Zeng, L., Lalgondar, M., Bevan, D., Winkel, B., Mohamed, A., Cheng, C. L., Shih, M. C., Poulton, J., and Esen, A. 2004. Functional genomic analysis of Arabidopsis thaliana glycoside hydrolase family 1. Plant Mol. Biol. 55:343-367.

Yamada, K., Hara-Nishimura, I., and Nishimura, M. 2011. Unique defense strategy by the endoplasmic reticulum body in plants. Plant Cell Physiol. 52:2039-2049.

Yamada, K., Nagano, A. J., Nishina, M., Hara-Nishimura, I., and Nishimura, M. 2008. NAI2 is an endoplasmic reticulum body component that enables ER body formation in Arabidopsis thaliana. Plant Cell 20: 2529-2540. 